\title{
Suppression of Hepatic CYP3A1/2 and CYP2C11 by \\ Cyclosporine is Not Mediated by Altering Growth Hormone \\ Levels
}

Shirley K. Lu, Shellie M. Callahan, Lane J. Brunner

Pharmaceutics Division, College of Pharmacy, The University of Texas at Austin,

Austin, TX 78712-1074 
JPET/2002/44776

\section{Running Title: GH levels not mediating factor in CsA suppression of CYP}

Address correspondence to: $\quad$ Lane J. Brunner, Ph.D.

College of Pharmacy, PHR 4.214E

The University of Texas at Austin

Austin, TX 78712-1074

Tel: (512) 471-0942

Fax: (512) 471-7474

E-mail: lane.brunner@mail.utexas.edu

Number of text pages: 19

Number of tables: 2

Number of figures: 5

Number of references: 30

Number of words in Abstract: 268

Number of words in Introduction: 557

Number of words in Discussion: 1,282

Abbreviations: CYP, cytochrome P450; CsA, cyclosporine; CsA V, cyclosporine vehicle GH, growth hormone; GH V, Growth hormone vehicle; HPLC, high-performance liquid chromatography; X-OHT, where X designates the regio- and stereochemistry of the testosterone hydroxylation metabolite (e.g., $6 \beta$-OHT denotes $6 \beta$-hydroxytestosterone). 
JPET/2002/44776

\begin{abstract}
Cyclosporine (CsA) suppresses drug metabolism by decreasing cytochrome $\mathrm{P} 450$ (CYP) enzyme levels in rat liver. Growth hormone $(\mathrm{GH})$ is known to pretranslationally regulate CYP expression. Thus, the suppression of CYP by CsA may involve GH as an intermediate. In order to address this question, we examined the effects of administering exogenous GH via twice daily subcutaneous injections and in conjunction with chronic subcutaneous CsA administration for 14 days on hepatic CYP expression. CsA alone decreased CYP3A1/2 and CYP2C11 significantly in a similar manner as previously found. When administered in the absence of CsA, GH also suppressed CYP3A1/2 and CYP2C11 protein levels as compared to $\mathrm{GH}$ vehicle. In the presence of $\mathrm{CsA}$, GH did not cause a further suppression of either CYP3A1/2 or CYP2C11 expression when compared to $\mathrm{CsA}$ treatment with $\mathrm{GH}$ vehicle. Testosterone in vitro catalytic assays confirmed that CsA and GH separately cause significant decreases in activity levels. Also, the concomitant administration of GH and CsA caused lowered productions of $16 \alpha-, 2 \alpha-$, $6 \beta-$, and $2 \beta-\mathrm{OHT}$ as compared to the administration of $\mathrm{GH}$ with $\mathrm{CsA}$ vehicle and as compared to the administration of $\mathrm{GH}$ vehicle with CsA.

This study shows that $\mathrm{GH}$ is a dominating factor over CsA in determining hepatic CYP expression and activity. In addition, CsA does not seem to be altering GH levels as a mediating event in suppressing CYP expression and activity. Since CsA given in combination with GH further suppressed CYP activity as compared with CsA given in combination with vehicle, this suggests that changes in hormonal status is likely to be one of the many factors that is responsible for the lack of a clear association between cyclosporine dosing and markers of toxicity.
\end{abstract}


Immune suppression has been an effective avenue of treatment for several conditions including preventing organ transplantation rejection and autoimmune disease. One potent immunosuppressive drug cyclosporine (CsA), a cyclic undecapeptide of fungal origin, is often used as the drug of choice following organ transplantation. CsA is mainly used for the prevention of allograft rejection and for the prevention of graftversus-host disease following a bone marrow transplant as well as for the treatment of arthritis (Tugwell et al., 1987). Although CsA is known to affect interleukins 3 and 4 (IL3, IL-4), tumor necrosis factor- $\alpha$ (TNF- $\alpha)$, and B cells, it primarily imparts its immunosuppressive action by preventing interleukin-2 (IL-2) synthesis from activated Tcells (Bunjes et al., 1981).

Despite the effectiveness of CsA in suppressing the immune response, there exist several potentially harmful side effects, including nephrotoxicity, hepatotoxicity, and hypertension (Borel, 1990). Since CsA is not only a substrate, but also an inhibitor of CYP3A2, CsA can modify hepatic drug metabolism in rats following chronic therapy (Brunner et al., 1996). CsA suppresses cytochrome P450 (CYP) protein expression, which could then hinder further metabolism of CsA (Cunningham et al., 1985; Brunner et al., 1996). This cycle results in an accumulation of CsA ultimately leading to organ toxicity. More specifically, it was found that CsA suppressed hepatic CYP3A1/2 and 2C11 in a time-dependent and dose-dependent manner in animals (Brunner et al., 1998; Brunner et al., 2000). Although this phenomenon has been readily demonstrated in the rat, CsA-induced changes in hepatic metabolism have yet to be identified clearly in humans. 
Pituitary hormones are known to play a significant role in CYP expression in mammals. Unlike other pituitary hormones, growth hormone (GH) lacks specific target sites. Because of this, GH can exert a wide range of physiological and metabolic effects on target tissues. GH alters drug metabolism in the liver by influencing the regulation of CYP expression. From as early as 1973, the regulation of hepatic drug metabolism enzymes in the rat was shown to be dependent on the amount of $\mathrm{GH}$ present in vivo (Wilson, 1973). In determining sex-specific expressions of drug-metabolizing enzymes, the pattern of GH secretion is more relevant than the actual amount of GH present. When $\mathrm{GH}$ is secreted in a pulsatile fashion, the male-specific CYP isoform CYP2C11, along with its corresponding steroid $16 \alpha$ - and $2 \alpha$-hydroxylase activities, predominates (Morgan et al., 1985; Waxman et al., 1991).Other CYP isoforms including CYP3A1/2, CYP2A2, and CYP2C13 are also male-specific, but may not necessarily depend directly on intermittent GH pulses (Waxman et al., 1988; Waxman et al., 1995). On the other hand, if $\mathrm{GH}$ is secreted in a continuous pattern, this is indicative of a female secretion pattern. In this case, CYP2C12 prevails, while levels of CYP2C11 and CYP3A1/2 are low to nonexistent (MacGeoch et al., 1985; Waxman et al., 1985). Since CYP enzymes account for the majority of drug metabolism that takes place in a rat, any alteration of these enzyme levels could have a profound impact on the extent of drug metabolism and possibly lead to adverse pharmacological effects.

The purpose of this study was to investigate the role of GH in the modulation of drug-metabolizing enzymes by CsA. This could provide additional insight into the role of hormones in the regulation of drug metabolism and provide a clearer understanding of the physiological interactions that mediate the effects of CsA on CYP enzymes. 
JPET/2002/44776

\section{Materials and Methods}

\section{Materials}

CsA was generously provided by Novartis (East Hanover, NJ, USA) in the form of Sandimmune $\mathrm{TM}^{\mathrm{TM}}$ oral solution. The original dosage form was diluted in commercially available olive oil and stored in amber bottles to limit light exposure. The CsA vehicle was the same commercially available olive oil. Purified rat GH was generously provided by the National Hormone \& Pituitary Program under the National Institute of Diabetes \& Digestive \& Kidney Diseases (NIDDK, Torrance, CA, USA) and solubilized in $0.01 \mathrm{M}$ $\mathrm{NaHCO}_{3}$. GH vehicle consisted of $0.01 \mathrm{M} \mathrm{NaHCO}$. The anesthetic mixture consisted of a 1:1:1 (v:v:v) ratio of ketamine $(100 \mathrm{mg} / \mathrm{ml})$, xylazine $(20 \mathrm{mg} / \mathrm{ml})$, and acepromazine (10 $\mathrm{mg} / \mathrm{ml}$ ). Ketamine and xylazine were purchased from Sigma Chemical Co. (St. Louis, MO, USA). Acepromazine was purchased from Fort Dodge Laboratories, Inc. (Fort Dodge, LA, USA). Rat CYP3A1/2 and CYP2C11 selective polyclonal antibodies were purchased from Gentest Corporation (Woburn, MA, USA). Rat CYP3A1/2 antibody was isolated from goats that were immunized with CYP3A2 purified from rat liver. Due to the polyclonal nature of the antiserum, two indistinguishable bands (CYP3A1 and CYP3A2) are detected and is therefore collectively referred to as CYP3A1/2. The microsomal standard used for the relative quantitation of CYP3A1/2 protein was composed of phenobarbital-treated rat liver microsomes. Rat CYP2C11 antibody is also polyclonal and raised in goats, however the cross reactivity with CYP2C13 can be readily distinguished from CYP2C11 based on mobility. CYP2C11 standard was composed of untreated male rat liver microsomes. The horseradish peroxidase-conjugated rabbit anti-goat (secondary CYP antibody) was purchased from ICN Pharmaceuticals, Inc. (Aurora, OH, USA). Rat 
growth hormone enzyme immunoassay (EIA) kits were obtained from Cayman Chemical Co. (Ann Arbor, MI, USA) and were manufactured by Spi-Bio (Massy Cedex, France). All urine and serum creatinine assay slides were manufactured by Johnson \& Johnson's Ortho-Clinical Diagnostics division (Rochester, NY, USA).

\section{Animals}

All procedures were approved by the Institutional Animal Care and Use Committee of The University of Texas at Austin and are in accordance with the guidelines established by the National Institutes of Health for the humane treatment of animals. Nine- to ten-week old, male Sprague Dawley rats were purchased from Harlan Sprague Dawley, Inc. (Indianapolis, IN, USA). During the study, all rats were kept in a twelve-hour light/dark cycle environment with free access to standard rat chow (Harlan, Indianapolis, IN, USA) and deionized water. Food consumption and body weight were measured daily.

Rats were randomly assigned to one of six groups ( $\mathrm{N}=6$ each). The groups are denoted: 14 CsA, 14 CsA V, 14 GH/CsA, 14 GH/CsA V, 14 GH V/CsA, and 14 GH V/CsA V (V = vehicle). Animals receiving GH were administered twice daily subcutaneous doses of $120 \mathrm{ng} / \mathrm{g}$ body weight of rat GH according to previous reports of successful supplementation of GH (Waxman et al., 1991). The GH vehicle was administered in the same fashion as $\mathrm{GH}$ at $1 \mathrm{ml} / \mathrm{kg}$. CsA administration consisted of once daily subcutaneous injections of a $15 \mathrm{mg} / \mathrm{kg}$ dose and CsA vehicle was also administered once daily at a concentration of $1 \mathrm{ml} / \mathrm{kg}$.

\section{Blood collection}


On the second to last day of dosing, all animals underwent jugular cannula implantation surgery to allow for passive blood collection according to the method of Waynforth \& Flecknell (Waynforth and Flecknell, 1992). On the last day of dosing for each group, rats were placed into standard rodent metabolic cages for urine collection. Following this 24-hour period, $0.1 \mathrm{ml}$ blood samples were collected once every 15 minutes for a six-hour period via the in-dwelling jugular cannula. After collection, the blood was allowed to clot on ice and was centrifuged at $9000 \mathrm{x} g$ for 5 minutes at $4^{\circ} \mathrm{C}$. Serum was harvested immediately following each spin and stored at $-80^{\circ} \mathrm{C}$ until time of assay.

\section{Microsome isolation}

Upon sacrifice of animals, the liver was immediately excised. Liver microsomal isolation was achieved with the use of a previously described method of differential centrifugation (Coon et al., 1978) and kept at $4{ }^{\circ} \mathrm{C}$ during the entire preparation. Liver tissue was ground in three volumes of Tris chloride buffer consisting of 0.1 M EDTA and 0.15 M potassium chloride using a PowerGen 700 homogenizer (Fisher Scientific, Pittsburgh, PA, USA). Samples were then centrifuged at $9,000 \mathrm{x} g$ for 20 minutes at $4{ }^{\circ} \mathrm{C}$. The supernatant was collected and centrifuged at 550,000 $\mathrm{x} g$ for 17 minutes at $4{ }^{\circ} \mathrm{C}$. The supernatant was discarded and the pellet resuspended in sodium pyrophosphate buffer containing 0.1 M EDTA at $\mathrm{pH}$ 7.4. The suspension was then homogenized and afterward centrifuged at 550,000 x $g$ for another 17 minutes at $4{ }^{\circ} \mathrm{C}$. The supernatant was discarded and the pellet washed and homogenized in Tris buffer containing $20 \%$ glycerol for storage. The microsomes were then stored at $-80{ }^{\circ} \mathrm{C}$ until analysis.

\section{Gel electrophoresis and immunoblot analysis}


Gel electrophoresis was performed using an SDS-PAGE $8 \%$ polyacrylamide separating gel as previously described (Laemmli, 1970). Protein on the gel was then transferred to nitrocellulose sheets by a prior described method (Schnier et al., 1989). After transfer of proteins, the nitrocellulose sheets were blocked with $3 \%$ non-fat dry milk (NFDM) in tris-buffered saline (TBS) at room temperature. Detection of putative proteins was achieved with goat anti-rat IgG (in a 1:2000 dilution) immunoreactive to the specific CYP enzyme of interest in 3\% NFDM and then rabbit anti-goat horseradish peroxidase (in a 1:2000 dilution) also in 3\% NFDM at room temperature. Transitional washes using TBS and $0.05 \%$ Tween 20 in TBS were done according to previously described procedures (Schnier et al., 1989). Immune complexes for CYP3A1/2 and CYP2C11 were detected with an NEN chemilumnescence reagent kit as described by the manufacturer (New England Nuclear Life Science Products, Boston, MA, USA). Blot densities were measured using a flatbed scanner (Microtek, Hsinchu, Taiwan) and analyzed on a Dell PC computer using the Kodak 1D image analysis software, version 3.5 (Eastman Kodak Co., Rochester, NY, USA).

\section{Testosterone Hydroxylation Assay and HPLC assay}

Liver samples for the testosterone hydroxylation assay were performed as previously described (Brunner et al., 1996). In brief, $200 \mu \mathrm{g}$ of liver microsomal protein was added to $0.02 \mathrm{M}$ potassium phophate buffer ( $\mathrm{pH} 7.4$ ), regeneration system, and water. The samples were then incubated with $250 \mu \mathrm{M}$ testosterone for 3 minutes and with glucose-6-phosphate dehydrogenase for 15 minutes under the same conditions. The reaction was quenched with dichloromethane and $11 \alpha$-hydroxyprogesterone was added 
as the internal standard. Once the organic phase was transferred and evaporated, they were dissolved in methanol and stored at $4^{\circ} \mathrm{C}$ for no longer than 1 month before use.

Separation and detection of testosterone and metabolites were performed on a Shimadzu HPLC system (Shimadzu Scientific Instruments, Inc., Columbia, MD, USA) equipped with an automatic injection system, dual solvent delivery pumps, a system controller, and a variable visual/ultraviolet wavelength detector. The column (LC-18) was kept at a constant temperature of $40^{\circ} \mathrm{C}$ and a wavelength of $238 \mathrm{~nm}$ was used to detect the analytes. Peak areas of corresponding hydroxylation metabolites were measured and compared to peak areas of the internal standard within the same run.

\section{Creatinine Clearance}

Serum creatinine was evaluated using Vitros creatinine DT slides on the Vitros DT60 II chemistry system (Rochester, NY, USA). The analysis is based on the hydrolysis of creatinine to form ammonia. Once ammonia is formed and creates a blue color, the intensity of the color is detected by a photodetector located within a fiber-optic reflection system and compared to a blank ammonia slide. The concentration of sample is calculated based on these measurements and the calibration measurements.

Urine creatinine measurements were calculated based on a similar system as the serum creatinine with the exception that all components were contained in one slide. Creatinine clearance was calculated as follows:

$$
\text { Clcr }=(\text { urine creatinine/serum creatinine }) \mathrm{X} \text { urine flow rate }
$$

Values were expressed in units of $\mu 1 / \mathrm{min} / 100 \mathrm{~g}$ body weight.

\section{rGH Enzyme Immunoassay}


The enzyme immunoassay used to determine GH levels was manufactured by Spi-Bio (Massy Cedex, France). The principle of the assay is based on the competition between unlabelled rat $\mathrm{GH}$ and acetylcholinesterase bound to rat growth hormone tracer. The yellow color formed by exposing the sample and acetylcholinesterase to an enzymatic substrate (and chromagen) for acetylcholinesterase was measured spectrophotometrically. The limit of detection for this assay was $0.5 \mathrm{ng} / \mathrm{ml}$.

\section{CsA Monoclonal Whole Blood Assay}

Levels of CsA in blood were measured using the TDxFLx ${ }^{\circledR}$ system (Abbott Laboratories, Abbott Park, IL, USA). The system uses fluorescence polarization immunoassay (FPIA) technology as the basis for detecting CsA in blood. In brief, the competitive binding assay involves a tracer-labeled antigen, antibody, and the sample blood. In reference to sensitivity, the CsA monoclonal whole blood assay can detect CsA levels with $95 \%$ confidence for samples containing $\geq 25 \mathrm{ng} / \mathrm{ml}$ of CsA. Assay precision was determined by Abbott Laboratories to be $<4 \% \mathrm{CV}$.

\section{Data Analysis}

Densities from Western blots were compared with standard microsomal proteins and expressed as a percentage. Mean peak quantity, mean peak amplitude, and mean peak duration determinations were calculated using the ULTRA analysis program (Van Cauter, 1988). In brief, the general principle of the program relies on the elimination of all peaks that do not meet the threshold requirements for a significant pulse. The measurements used are based on a comparison of the increment of the preceding nadir and the decrement of the preceding peak to a multiple of the intra-assay coefficient. Pulses that do not exceed the threshold criteria are eliminated from the series. One-way 
ANOVA and a priori means comparisons tests were employed using respective vehicle groups as the control with the aid of the SuperANOVA statistical program (Abacus Concepts, Inc., Berkeley, CA, USA). Data are presented as mean \pm standard error. When the probability of chance explaining the results was reduced to less than $5 \%(\mathrm{p}<0.05)$, the differences were then considered to be statistically significant. 
JPET/2002/44776

\section{Results}

\section{Effect of concomitant administration of rGH and CsA on hepatic CYP levels}

Figure 1 represents hepatic CYP3A1/2 and CYP2C11 protein expression after 14 days of dosing as determined by Western blotting. The results from rats treated for 14 days revealed a significant decrease in CYP3A1/2 and CYP2C11 levels in the CsA group as compared to the vehicle control group ( $\mathrm{p}=0.04 ; \mathrm{p}=0.004$, respectively), which is in agreement with previous reports from our lab (Brunner et al., 2000). Similarly, when CsA was administered in combination with GH vehicle, both isoforms were markedly depressed when compared with $\mathrm{GH}$ vehicle/CsA vehicle treatment. When exogenous $\mathrm{GH}$ was given with CsA, levels of CYP3A1/2 and CYP2C11 were not suppressed as compared to the GH/CsA vehicle group. Additionally, when administered concomitantly with CsA vehicle, excess GH caused a significant decrease in CYP3A1/2 and CYP2C11 expression $(\mathrm{p}=0.01 ; \mathrm{p}=0.007$, respectively).

\section{Effect of concomitant administration of rGH and CsA on hepatic CYP activity measured by testosterone hydroxylation assay and HPLC}

The formation of $6 \beta$-hydroxytestosterone ( $6 \beta-\mathrm{OHT})$ correlates primarily with the activity of CYP3A2 (Waxman et al., 1983; Waxman et al., 1985), and to a lesser extent with CYP3A1 (Sonderfan et al., 1987). HPLC analysis following testosterone hydroxylation assay of liver microsomes showed a significant decrease in the production of $6 \beta-\mathrm{OHT}$ ( $\mathrm{p}=0.0001)$ in the group receiving CsA only as compared to the group receiving CsA vehicle only, indicating a reduction in the overall activity of CYP3A1/2 (figure 2). This finding supports the suppression in protein levels found with Western blotting. Significantly lower quantities of $6 \beta-\mathrm{OHT}$ were detected in all groups 
administered $\mathrm{GH}(\mathrm{GH} / \mathrm{CsA} \mathrm{V}, \mathrm{GH} / \mathrm{CsA})$ versus the respective $\mathrm{GH}$ vehicle groups $(\mathrm{p}=0.026 ; \mathrm{p}=0.0001)$, thus confirming that superphysiological expression of $\mathrm{GH}$ decreases CYP3A1/2 activity.

The formation of $16 \alpha-\mathrm{OHT}$ and $2 \alpha-\mathrm{OHT}$ correspond to the activity of CYP2C11 (Cheng and Schenkman, 1983; Waxman, 1984). Since the pattern of results was nearly identical for the formation of these two metabolites, only the results for $2 \alpha-\mathrm{OHT}$ are represented in graphical form. Following 14 days of treatment with CsA, activity levels of CYP2C11 were lowered significantly as compared with CsA vehicle treatment. CsA treatment alone and in conjunction with GH vehicle also dramatically decreased the formation of $2 \alpha-\mathrm{OHT}(\mathrm{p}=0.0001 ; \mathrm{p}=0.0001$, respectively), as well as $16 \alpha-\mathrm{OHT}$ $(\mathrm{p}=0.0001 ; \mathrm{p}=0.0001$, respectively). Interestingly, administration of $\mathrm{GH}$ (as compared to GH vehicle) significantly lowered the formations of $2 \alpha-$ and $16 \alpha$ - OHT, irrespective of the concomitant drug (CsA or CsA vehicle).

\section{Effect of concomitant administration of $\mathrm{rGH}$ and CsA on renal function parameters}

Urine volume collected over the 24-hour period was compared as a measure of kidney function (table 1). CsA-treated rats had a nearly 2-fold greater volume of urine output as compared with the CsA vehicle-treated rats $(\mathrm{p}=0.02)$. Similarly, the CsAtreated rats given $\mathrm{GH}$ vehicle had a 2.3-fold increase in urine volume when compared with CsA-treated rats given $\mathrm{GH}$ vehicle. However, when CsA-treated rats were administered $\mathrm{GH}$, the urine flow volume was significantly decreased from the CsA/GH vehicle group $(\mathrm{p}=0.038)$.

Serum creatinine levels and creatinine clearance rates were analyzed as an estimation of glomerular filtration rate (table 1). Serum creatinine was only slightly 
higher in the $\mathrm{GH} / \mathrm{CsA}$ group as compared with the $\mathrm{GH} / \mathrm{CsA}$ vehicle group ( $\mathrm{p}=0.05)$. No other differences in serum creatinine levels were detected for any of the other groups. In addition, no significant differences in creatinine clearance, due to either administration of $\mathrm{GH}$ or CsA, were detected. 
JPET/2002/44776

\section{Circulating serum growth hormone levels}

Figure 3 shows the area under the curve (AUC) for all groups. The groups that were administered two injections a day (regardless of the agents injected) generally had higher AUCs due to the combination of both higher frequency of pulses and a higher basal level of secretion. No statistically significant differences were observed between respective treatment groups. Representative six-hour GH secretion profiles from each group are presented in figure 4. GH release profile parameters including mean peak amplitude, mean peak number, and mean peak duration are shown in table 2 . No statistically significant differences were detected between treatment groups and their respective controls.

\section{CsA Blood Levels}

The concentration of CsA in blood for animals administered GH and CsA was

slightly higher, but not statistically different from animals injected with GH vehicle and CsA ( $\mathrm{p}=0.175)$. This was the only comparison performed since no other comparisons were useful from a statistical standpoint. 
JPET/2002/44776

\section{Discussion}

The primary aim of the present study was to determine if GH is the main intermediate through which the chronic suppression of CYP by CsA occurs in the liver of rats. With the pituitary intact, the GH secretory profile is still present and suppression of normal levels of CYP enzymes can be readily detected. The relationship between CsA, $\mathrm{GH}$, and CYP enzymes was examined by introducing exogenous GH to intact male rats while concomitantly administering CsA. Select CYP isoforms' expressions and activities were analyzed as indicators of drug metabolism.

Because CYP3A1/2 contributes significantly to the metabolism of numerous xenobiotics (including CsA) that takes place in the rat liver and CsA is known to suppress this isoform, the expression and behavior of this isoform was of chief importance for this study. Previous studies show that when exogenous GH is introduced to intact male rats, CYP3A2 is significantly suppressed (Kawai et al., 2000; Kawai et al., 2001). Our study confirmed that 14-day treatment with $\mathrm{GH}$ suppresses CYP3A1/2 protein expression. However, when CsA was administered in combination with GH, GH did not cause a significant suppression of CYP3A1/2 protein as compared to concomitant administration of CsA with $\mathrm{GH}$ vehicle. This masking effect was also evident when CsA failed to cause suppression when administered with $\mathrm{GH}$ and compared to administration with $\mathrm{GH}$ vehicle. Also, all groups receiving two injections per day experienced an overall decline in CYP3A1/2 protein expression, irrespective of the drug. This finding corresponds with the trend in GH levels represented by AUC calculations in this study. GH levels for groups receiving two injections were generally higher than groups receiving only one. This could partly be due to the stress involved with the injection and is therefore 
unrelated to the drug admininistered. A previous study revealed a significant increase in GH following 10-11 days of only saline injection in adult male rats (Kant et al., 1983).

Since CYP3A1/2 catalyzes the production of both $6 \beta-\mathrm{OHT}$ and $2 \beta-\mathrm{OHT}$, the quantities of both derivatives were measured to signify CYP3A1/2 activity. We showed that after 14 days of dosing, exogenous rat GH administered to normal rats suppressed both $6 \beta$ - and $2 \beta$-OHT production. This result was evident despite co-administration with either CsA or CsA vehicle. Similarly, a previous study has reported depression of $6 \beta$ OHT levels after 7 days in intact male rat given GH in both intermittent pulses as well as in a continuous infusion (Yamazoe et al., 1986). Because the continuous presence of GH has been shown to decrease CYP3A2 activity in male rats and the long-term intermittent pulses used for this study resulted in a decrease in the activity of CYP3A1/2, this indicates that CYP3A1/2 may be responsive to an overall chronic increase in circulating $\mathrm{GH}$ levels. The data also show that $\mathrm{GH}$ and $\mathrm{CsA}$ have an additive suppressive effect on CYP3A1/2 activity as compared to CsA's suppressive effects alone. Since CYP3A2 is the main isoform responsible for the metabolism of CsA, the considerable decline in CYP3A1/2 activity as a result of CsA and GH combined will likely lead to higher concentrations of CsA in circulation, thus leading to increased incidence of organ toxicity.

CYP2C11 is a sex-specific isoform whose expression is most directly regulated by the pulsatile secretions of the male GH pattern (Waxman et al., 1991; Legraverend et al., 1992). This concept was further validated when pulsatile secretions of GH via an external syringe pump as well as subcutaneous injections replaced the male-pattern GH secretions in hypophysectomized male rats and resulted in an increase in CYP2C11 
mRNA expression, as well as $2 \alpha-\mathrm{OHT}$ activity (Waxman et al., 1991). Although twice daily subcutaneous injections of GH is known to increase CYP2C11 to normal levels in hypophysectomized rats, we show that administration of GH in the same fashion to intact rats causes a decrease in $\mathrm{CYP} 2 \mathrm{C} 11 . \mathrm{GH}$ administration (with CsA vehicle) significantly reduced CYP2C11 expression when compared with the corresponding GH vehicle group. Nearly the same result has also been obtained by other researchers using the same route of administration, dose, and a comparable length study period of 12 days (Kawai et al., 2001). This could indicate that exogenous GH, in addition to endogenously secreted $\mathrm{GH}$, is causing the animals to continually have $\mathrm{GH}$ present in circulation, effectively increasing the basal level of GH. The immediate downstream consequence of the substantially higher concentration of $\mathrm{GH}$ in plasma may be that the $\mathrm{GH}$ receptor is not functioning in the correct capacity to initiate the JAK/STAT pathway. This notion is supported by the GH receptor dimerization theory, whereby GH receptors only function to trigger the intracellular signal transduction necessary for initiation of CYP protein gene transcription when they are bound to one GH molecule and then dimerize with another GH receptor molecule (Fuh et al., 1992). Therefore, excess GH in circulation may lead to a 1:1 ratio of binding and would not allow for the 1:2 ratio of $\mathrm{GH}: \mathrm{GH}$ receptor necessary for initiation of action by $\mathrm{GH}$.

A significant decrease in the production of $2 \alpha-\mathrm{OHT}$ and $16 \alpha-\mathrm{OHT}$ was exhibited with the administration of CsA alone as compared to the administration of CsA vehicle alone. This is consistent with previous results showing a suppression of CYP2C11 protein expression and enzyme activity as a result of chronic CsA administration (Brunner et al., 1996). The administration of CsA in combination with GH resulted in a 
further suppression of CYP2C11 activity than when either agent was administered with vehicle. Although this is in slight discordance with protein levels, the trend appears similar although the results were not statistically significant for the protein expression data. Considering that CYP2C11 is the predominant CYP enzyme present in male rat liver, the additive suppressive effect of combined GH and CsA therapy on CYP2C11 activity may have profound consequences on drug metabolism overall.

GH secretion profile parameters were not significantly altered due to CsA treatment, which revealed that chronic CsA treatment does not alter the level of GH in circulation. Since modifying the levels of GH can lead to a change in CYP3A2 and CYP2C11 levels, CsA does not seem to be affecting CYP expression by modulating GH levels. GH may still mediate the suppression of CYP enzymes by CsA at the receptorbinding or signal transduction level. Conversely, GH did not have an effect on detectable CsA concentrations in blood either. Because the combined administration of CsA and $\mathrm{GH}$ produced significantly lower CYP3A2 activity than when CsA was given with vehicle, we expected to see a considerably higher concentration of CsA in blood as a result of decreased CsA metabolism by CYP3A2. However, only a slight elevation was observed. This may be due to CsA being sequestered into organs, including the liver, kidney, and small intestine, thus preventing detection in blood.

The data also suggest that changes in hormonal status is likely to be one of the many factors that is responsible for the lack of a clear association between cyclosporine dosing and markers of toxicity. Hormonal status can be altered by either manipulating the amount of hormone in circulation or the subsequent signaling action of the hormone. Modulation of GH action can occur by one or more of several possible methods including 
a modification of the binding capacity of circulating $\mathrm{GH}$, alteration of $\mathrm{GH}$ receptor (GHR) quantity or binding capability and/or availability, alteration of Janus kinase-2 (JAK-2) action, or modification of any one of the GH-activated intracellular signaling pathways (Frank et al., 2000). According to this rationale, an alteration anywhere along the GH activation pathway could affect intracellular signaling and eventually, the downstream gene regulation of CYP protein production. Future studies are warranted to investigate GH binding and the signal transduction mechanism behind the possible function of GH in the alteration of CYP expression and activity by CsA. 


\section{Acknowledgements}

We would like to thank Dr. Eve Van Cauter and Rachel Leproult for graciously providing a copy of the ULTRA analysis software program. 


\section{References}

Borel J (1990) Pharmacology of cyclosporine (sandimmune). IV. Pharmacological properties in vivo. Pharmacol Rev 41:259-371.

Brunner L, Bennett W and Koop D (1996) Selective suppression of rat hepatic microsomal activity during chronic cyclosporine nephrotoxicity. J Pharmacol Exp Ther 277:1710-1718.

Brunner L, Bennett W and Koop D (1998) Cyclosporine suppresses rat hepatic cytochrome P450 in a time-dependent manner. Kid Intl 54:216-223.

Brunner L, Werner U and Gravenall C (2000) Effect of dose on cyclosporine-induced suppression of hepatic cytochrome P450 3A2 and 2C11. Eur J Pharmacol Biopharm 49:129-135.

Bunjes D, Hardt C, Rollinghoff M and Wagner H (1981) Cyclosporin A mediates immunosuppression of primary cytotoxic $\mathrm{T}$ cell responses by impairing the release of interleukin 1 and interleukin 2. Eur J Immunol 11:657-661.

Cheng K and Schenkman J (1983) Testosterone metabolism by cytochrome P-450 isozymes RLM3 and RLM5 and by microsomes: Metabolite identification. J Biol Chem 258:11738-11744.

Coon M, Hoeven Tvd, Dahl S and Haugen D (1978) Two forms of liver microsomal cytochrome P-450, P-450 LM2, and P-450 LM4. Meth Enzym 52:109-117.

Cunningham C, Burke M, Wheatley D, Thomson A, Simpson J and Whiting P (1985) Amelioration of cyclosporin-induced nephrotoxicity in rats by induction of hepatic drug metabolism. Biochem Pharmacol 34:573-587. 
Frank S, Messina J, Baumann G, Black R and Bertics P (2000) Insights into modulation of (and by) growth hormone signaling. J Lab \& Clin Med 136:14-20.

Fuh G, Cunningham B, Fukunaga R, Nagata S, Goeddel D and Wells J (1992) Rational design of potent antagonists to the human growth hormone receptor. Science 256:1677-1680.

Kant G, Bunnell B, Mougey E, Pennington L and Meyerhoff J (1983) Effects of repeated stress on pituitary cyclic AMP, and plasma prolactin, corticosterone and grwoth hormone in male rats. Pharmacol Biochem \& Behav 18:967-971.

Kawai M, Bandiera S, Chang T and Bellward G (2000) Growth hormone regulation and developmental expression of rat hepatic CYP3A18, CYP3A9, and CYP3A2. Biochem Pharmacol 59:1277-1287.

Kawai M, Bandiera S, Chang T and Bellward G (2001) Effect of exogenous growth hormone on somatic growth, gonadal development, and hepatic CYP2C11 and CYP2C12 expression in prepubertal intact male rats. Can J Physiol Pharmacol 79:352-361.

Laemmli U (1970) Cleavage of structural proteins during the assembly of the head of bacteriophage T4. Nature 227:680-685.

Legraverend C, Mode A, Westin S, Strom A, Eguchi H, Zaphiropoulos P and Gustafsson J-A (1992) Transcriptional regulation of rat P-450 2C gene subfamily members by the sexually dimorphic pattern of growth hormone secretion. Mol Endocrinol 6:259-266. 
MacGeoch C, Morgan E and Gustafsson J-A (1985) Hypothalamo-pituitary regulation of cytochrome P-450 15b apoprotein levels in rat liver. Endocrinology 117:20852092.

Morgan E, MacGeoch C and Gustafsson J-A (1985) Hormonal and developmental regulation of expression of the hepatic micorsomal steroid 16a-hydroxylase cytochrome P-450 apoprotein in the rat. J Biol Chem 260:11895-11898.

Schnier G, Laethem C and Koop D (1989) Identification and induction of cytochromes P450, P450IIE1, and P-450 IA1 in rabbit bone marrow. J Pharmacol Exp Ther 251:790-796.

Sonderfan A, Arlotto M, Dutton D, McMillen S and Parkinson A (1987) Regulation of testosterone hydroxylation by rat liver microsomal cytochrome P-450. Arch Biochem Biophys 255:27-41.

Tugwell P, Bombardier C, Gent M, Bennett K, Ludwin D, Grace E, Buchanan W, Bensen W, Bellamy N, Murphy G and Graffenried Bv (1987) Low-dose cyclosporine in rheumatoid arthritis: a pilot study. J Rheumatol 14:1108-1114.

Van Cauter E (1988) Estimating false-positive and false-negative errors in analyses of hormonal pulsatility. Amer J Physiol 254:E786-794.

Waxman D (1984) Rat hepatic cytochrome P-450 isoenzyme 2c. J Biol Chem 259:1548115490.

Waxman D, Dannan G and Guengerich F (1985) Regulation of rat hepatic cytochrome P450: Age-dependent expression, hormonal imprinting, and xenobiotic inducibility of sex-specific isoenzymes. Biochemistry 24:4409-4417. 
Waxman D, Ko A and Walsh C (1983) Regioselectivity and stereoselectivity of androgen hydroxylations catalyzed by cytochrome P-450 isozymes purified from phenobarbital-induced rat liver. J Biol Chem 258:11937-11947.

Waxman D, LeBlanc G, Morrissey J, Staunton J and Lapenson D (1988) Adult malespecific and neonatally programmed rat hepatic P-450 forms RLM2 and 2a are not dependent on pulsatile plasma growth hormone for expression. $J$ Biol Chem 263:11396-11406.

Waxman D, Pampori N, Ram P, Agrawal A and Shapiro B (1991) Interpulse interval in circulating growth hormone patterns regulates sexually dimorphic expression of hepatic cytochrome P450. Proc Natl Acad Sci 88:6868-6872.

Waxman D, Ram P, Pampori N and Shapiro B (1995) Growth hormone regulation of male-specific rat liver P450s 2A2 and 3A2: Induction by intermittent growth hormone pulses in male but not female rats rendered growth hormone deficient by neonatal monosodium glutamate. Mol Pharmacol 48:790-797.

Waynforth $\mathrm{H}$ and Flecknell $\mathrm{P}$ (1992) Experimental and surgical technique in the rat. Academic Press Inc., San Diego.

Wilson J (1973) Growth hormone modulation of liver drug metabolic enzyme activity in the rat. Specificity of the hormone effect. Proc Soc Exp Biol Med 143:978-985. Yamazoe Y, Shimada M, Murayama N, Kawano S and Kato R (1986) The regulation by growth hormone of microsomal testosterone 6 beta-hydroxylase in male rat livers. J Biochem 100:1095-1097. 
JPET/2002/44776

\section{Footnotes}

This research was supported by a grant from the National Institute of General Medical

Sciences (GM 60910). Data from this manuscript was previously presented at the 2002

American Association of Pharmaceutical Scientists meeting in Toronto, Canada. Please

direct reprint requests to:

Lane J. Brunner

The University of Texas at Austin

College of Pharmacy

1 University Station A1920

Austin, TX 78712-1074 
JPET/2002/44776

\section{Legends for Figures and Tables}

Figure 1 Western blot analysis of hepatic CYP3A1/2 and CYP2C11 microsomal protein expression in 14-day groups. Groups were given subcutaneous doses of either 15 $\mathrm{mg} / \mathrm{kg} \mathrm{CsA}$ or CsA vehicle alone or a combination of $\mathrm{GH}$, GH vehicle, CsA, and CsA vehicle. All values are expressed as a percent density of the protein standard used for each blot. $\mathbf{a}=\mathrm{p}<0.05$ between $\mathrm{C}$ and $\mathrm{CV}$ groups, $\mathbf{d}=\mathrm{p}<0.05$ between $\mathrm{G} / \mathrm{CV}$ and $\mathrm{GV} / \mathrm{CV}$ groups, $\mathbf{e}=\mathrm{p}<0.05$ between $\mathrm{GV} / \mathrm{C}$ and $\mathrm{GV} / \mathrm{CV}$ groups.

Figure 2 In vitro testosterone hydroxylation by hepatic microsomes following 14 days of treatment. Groups were given subcutaneous doses of either $15 \mathrm{mg} / \mathrm{kg} \mathrm{CsA}$ or CsA vehicle alone or a combination of $\mathrm{GH}, \mathrm{GH}$ vehicle, CsA, and CsA vehicle. Top graph represents $6 \beta$-hydroxylase activity; bottom graph represents $2 \alpha$-hydroxylase activity. Units for metabolite production are pmol metabolite/min/mg microsomal protein. $\mathrm{a}=\mathrm{p}<0.05$ between $\mathrm{C}$ and $\mathrm{CV}$ groups; $\mathrm{c}=\mathrm{p}<0.05$ between $\mathrm{G} / \mathrm{C}$ and $\mathrm{GV} / \mathrm{C}$ groups; $\mathrm{d}=\mathrm{p}<0.05$ between $\mathrm{G} / \mathrm{CV}$ and $\mathrm{GV} / \mathrm{CV}$ groups; $\mathrm{e}=\mathrm{p}<0.05$ between $\mathrm{GV} / \mathrm{C}$ and $\mathrm{GV} / \mathrm{CV}$ groups.

Figure 3 Area under the curve calculations of GH secretory profiles for circulating serum GH. Units are expressed as ng of circulating GH per ml of serum.

Figure 4 Representative graphs depicting GH secretion profiles of aforementioned groups over a 6-hour period. Blood samples were collected every 15 minutes. Figure 5 Representative blots for liver 3A1/2 and 2C11 protein expression, respectively. One representative animal was selected from each group for the densitometry figure. From left to right, the groups are: 14C, 14CV, 14G/C, 14G/CV, 14GV/C, 14GV/CV. 
Table $1 * * \mathrm{P}<0.05$ as compared to the respective CsA vehicle control group $\dagger=\mathrm{P}<0.05$ as compared to the respective $\mathrm{GH}$ vehicle control group

Table 2 No statistically significant differences were found between drug treatment groups and respective vehicle groups. 


\section{Table 1 Renal Function Parameters}

\begin{tabular}{|c|c|c|c|c|c|}
\hline Parameter & $\mathrm{CsA}$ & CsA V & $\mathrm{GH} / \mathrm{CsA}$ & $\mathrm{GH} / \mathrm{CsA} \mathrm{V}$ & 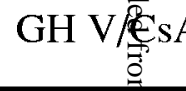 \\
\hline Weight Change $(\%)$ & $5.6 \pm 2.3^{*}$ & $10.8 \pm 1.6$ & $7.4 \pm 1.8^{*}$ & $19.7 \pm 0.6$ & 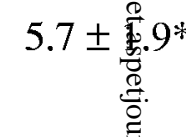 \\
\hline Urine Volume (ml) & $17.5 \pm 2.0^{*}$ & $9.0 \pm 2.1$ & $11.0 \pm 1.4^{\dagger}$ & $12.2 \pm 2.8$ & 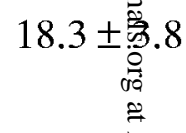 \\
\hline $\operatorname{Scr}(\mathrm{mg} / \mathrm{dl})$ & $0.66 \pm 0.04$ & $0.60 \pm 0.03$ & $0.76 \pm 0.07^{*}$ & $0.58 \pm 0.06$ & $0.67 \pm \tilde{7}$ \\
\hline $\operatorname{Clcr}(\mu \mathrm{l} / \mathrm{min} / 100 \mathrm{~g})$ & $283 \pm 53$ & $391 \pm 37$ & $268 \pm 29$ & $340 \pm 28$ & $418+\frac{\tilde{n}}{0}$ \\
\hline
\end{tabular}


Table 2 GH Release Profile Parameters

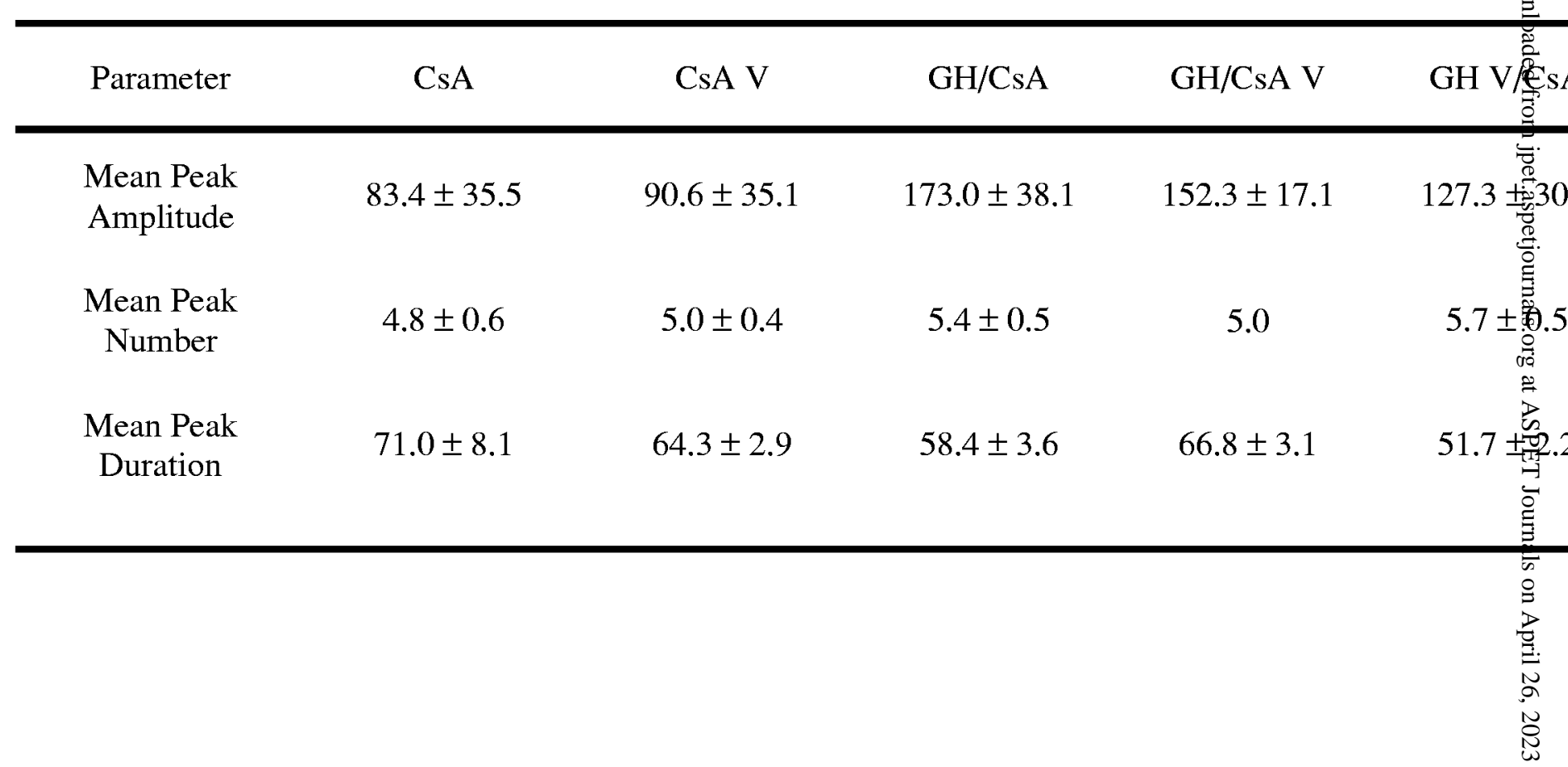


Figure 1 Suppressive effects of combined GH and CsA treatment on CYP3A1/2 and CYP2C11 expression are not additive
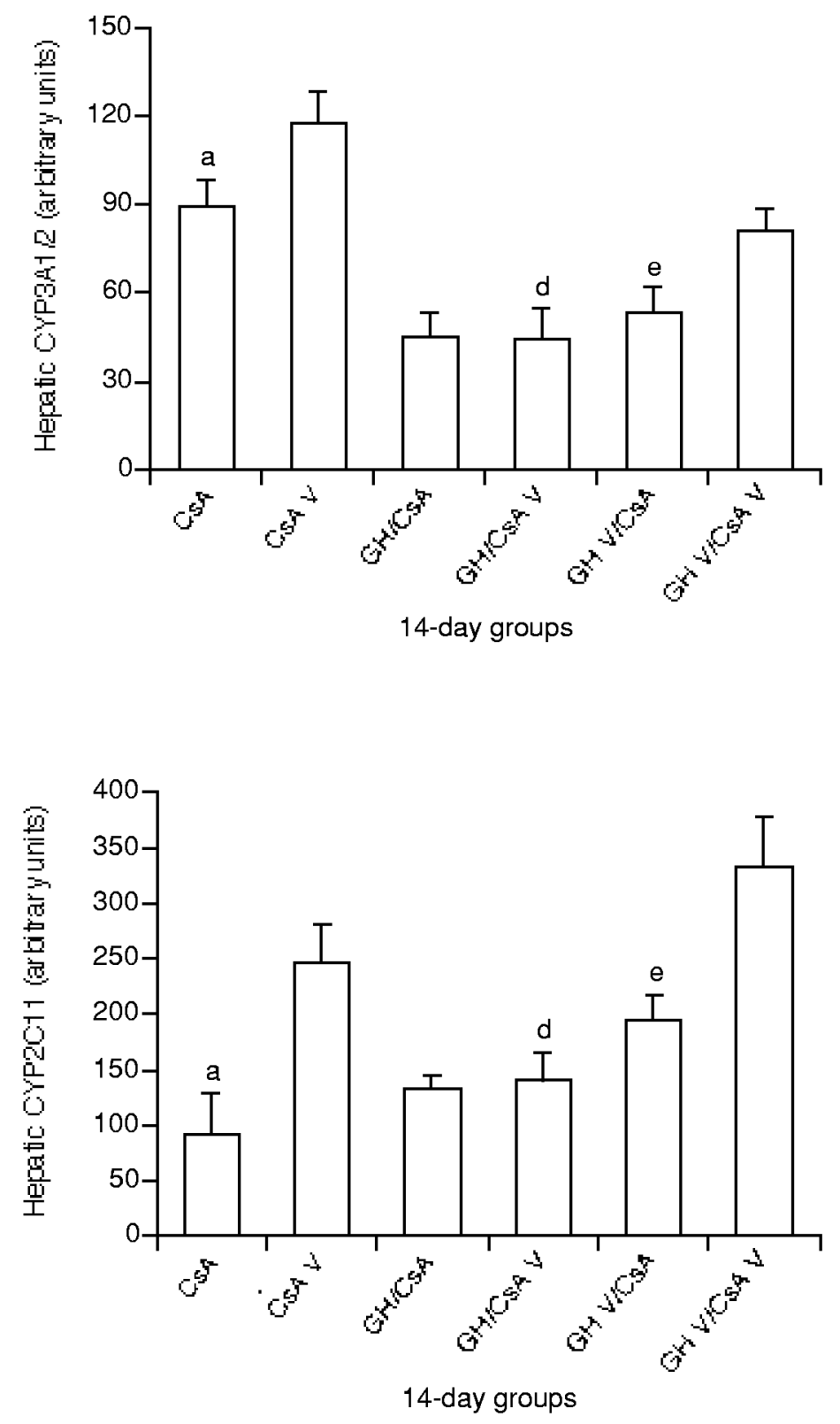
Figure 2 The suppressive effects of GH prevail over the suppressive effects of CsA on CYP3A1/2 and CYP2C11 activity
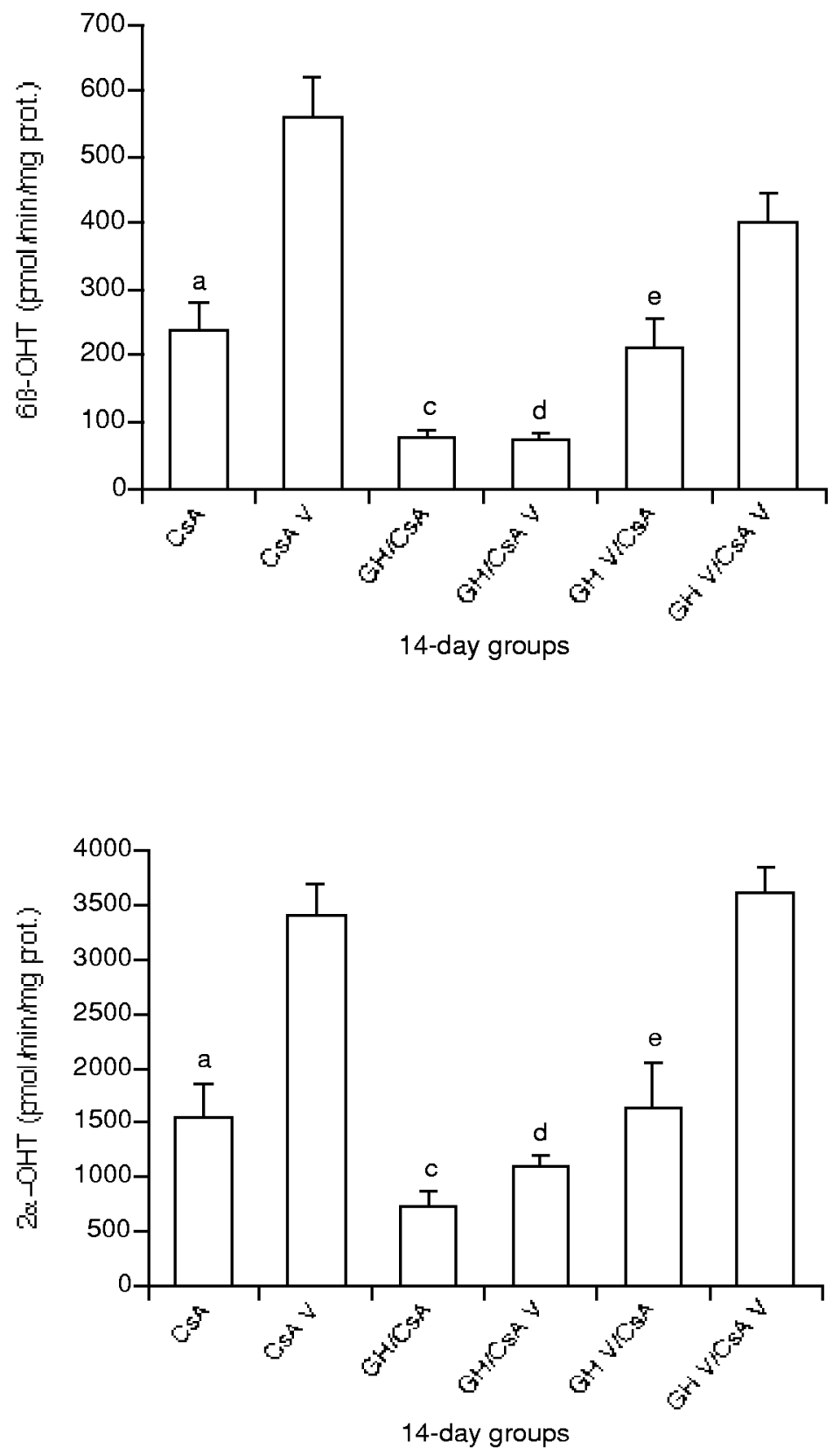
Figure 3 CsA did not significantly alter circulating GH levels and patterns

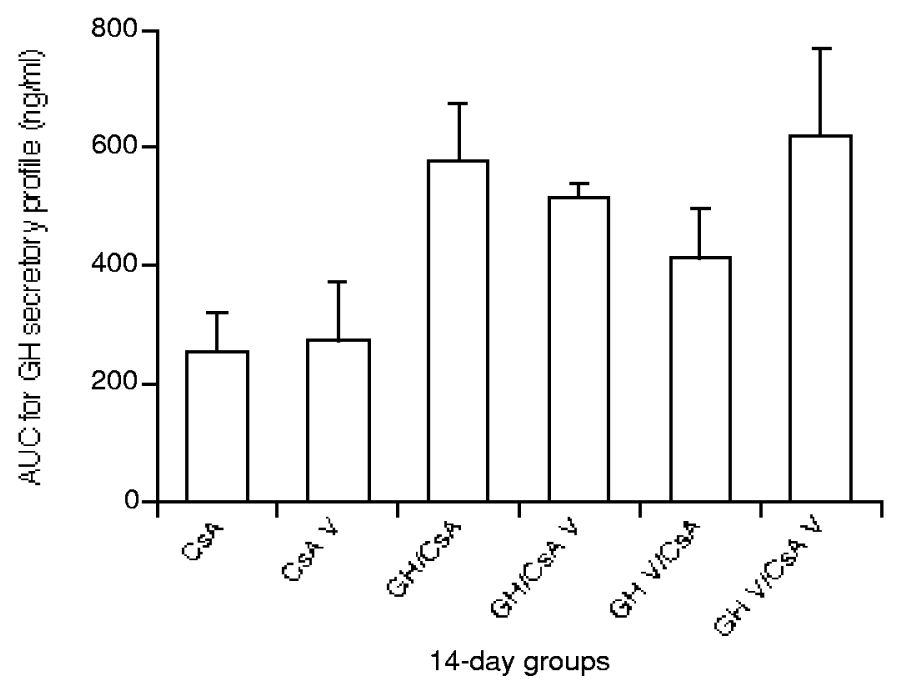




\section{Figure 4 Representative graphs of GH secretory profiles}
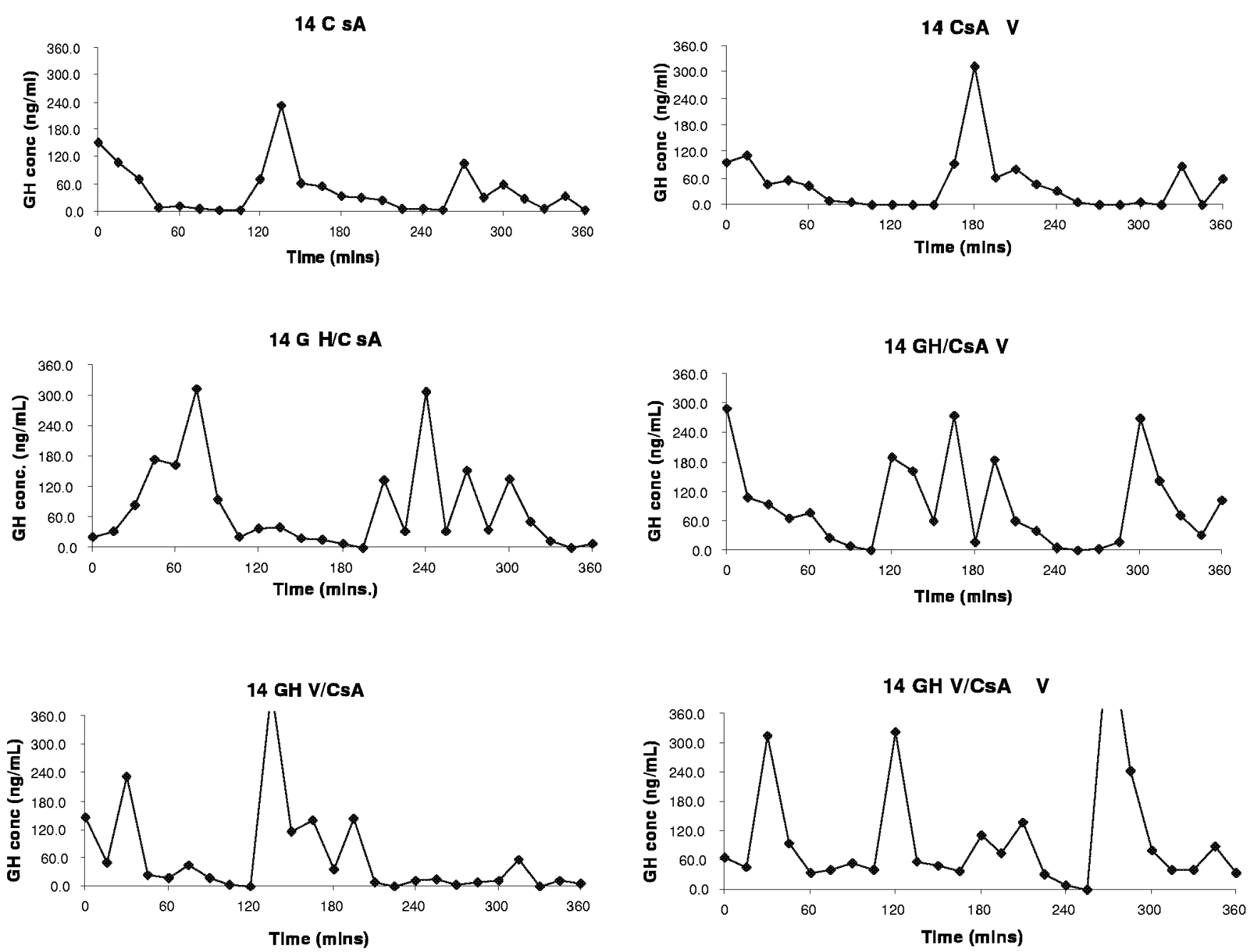


\section{Figure 5 Western blot densitometry}

\section{Liver $3 A 1 / 2$ representative blot}

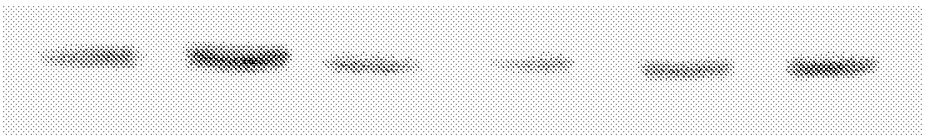

\section{Liver 2 C11 representative blot}

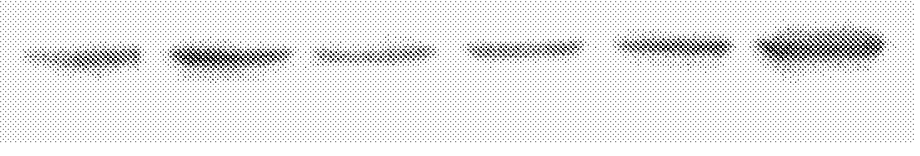

